\title{
Detection of collapsed and ordered polymer structures by fluorescence resonance energy transfer in stiff homopolymers: Bimodality in the reaction efficiency distribution
}

\author{
Goundla Srinivas and Biman Bagchi* \\ Solid State and Structural Chemistry Unit, Indian Institute of Science, Bangalore, India 560012
}

\begin{abstract}
Distribution of fluorescence resonance energy transfer (FRET) efficiency between the two ends of a stiff homopolymer chain is calculated by Brownian dynamics simulations. We show that the various collapsed and/or ordered conformations (spherical, rod, and toroidal) of a stiff polymer chain can be effectively distinguished by using the fluorescence resonance energy transfer technique. In particular, FRET efficiency distribution is unimodal at low chain stiffness (where collapsed structure is disordered) but becomes bimodal with increasing chain stiffness, when ordered structures are formed. We have also presented a simple theoretical analysis for the evaluation of the structural parameters.
\end{abstract}

\section{INTRODUCTION}

The structures of a collapsed homopolymer are a subject of much current interest. ${ }^{1-12}$ In particular, the effect of chain stiffness on the collapse transition has been extensively studied by Noguchi and Yoshikawa, ${ }^{2,7}$ by performing multicanonical Brownian dynamics simulations. ${ }^{13}$ They found that a semiflexible polymer exhibits various structures such as a rod, toroid, and also a fused structure between a toroid and a rod. They have also reported that in the case of chains with intermediate stiffness, the coil state changes first into a state in which rod and toroid shapes coexist. In the long time, only the rod structure survives at this intermediate stiffness. At high stiffness, only the toroid structure is stable.

The free energies of these structures are widely separated from one another, ${ }^{14}$ so these structures can be considered as representative of true minima of the free-energy surface. The existence of the above-mentioned structures has been confirmed by using various models such as the offlattice circular chain model by Kuznestov et al. ${ }^{3}$ and the bond-fluctuation model by Ivanov et al..$^{4,5}$ Although aspects such as the kinetic path from coiled structure to either toroid or the rod structures are still unclear, the morphological variation in a homopolymer chain has been well studied. A complete phase diagram representing the transition between various structural morphology of homopolymer chain has also been reported by Noguchi and Yoshikawa. ${ }^{2}$

In a notable recent development, fluorescence resonance energy transfer (FRET) has been combined with the advances in single molecule spectroscopic (SMS) technique ${ }^{15}$ to provide a powerful, novel approach to study the dynamics of polymers and proteins. ${ }^{16-21}$ Deniz et al. ${ }^{16}$ reported studies of dynamics of protein folding by observing FRET in the time domain from a single donor-acceptor (D-A) pair. Winnik and co-workers ${ }^{8,9}$ carried out a critical evaluation of di-

*Electronic mail: bbagchi@sscu.iisc.ernet.in rect energy transfer as a tool for analysis of nanoscale morphologies in polymers in block copolymer interfaces.

While the age-old paradigm in polymer chemistry has been that a polymer chain assumes conformation dictated by the random walk of its chain segments, recent single molecule spectroscopic studies by $\mathrm{Hu}$ et al. ${ }^{1}$ and simultaneous theoretical analysis have shown that structures of collapsed polymers in thin films are defective cylinders. These novel structures arise as a result of a competition between chain stiffness and chemical defects along the chain. Similar studies in the solution phase have not yet been carried out experimentally, although simulations have shown that the ordered structures are highly likely at high chain stiffness.

There is, however, no clear experimental approach yet to detect these alternate structures. In this study, we explore the possibility whether the fluorescence resonance energy transfer (FRET) between a donor and an acceptor chromophore embedded at the two ends of a polymer chain can serve as a probe to identify and study the structural morphology of the polymer chain. We find that FRET can indeed differentiate between the disordered collapsed state and the ordered states such as toroid or rod. This can be achieved by measuring the FRET efficiency distribution. The recent study of Deniz et al. ${ }^{16}$ demonstrated that the single molecular spectroscopy can be used to obtain the FRET efficiency distribution.

The usually assumed mechanism for FRET is the Förster energy transfer (FET). ${ }^{22,23}$ The rate of this transfer depends on the separation $(R)$ between the energy donor and the energy acceptor. This rate, $k(R)$, can be written as ${ }^{22}$

$$
k(R)=k_{\text {rad }}\left(\frac{R_{F}}{R}\right)^{6},
$$

where $R_{F}$ is the Förster radius and $k_{\text {rad }}$ is the radiative rate, which is typically in the range $10^{8}$ to $10^{9} \mathrm{~s}^{-1}$ for the commonly used chromophores. According to the above expression, the rate of energy transfer becomes equal to $k_{\text {rad }}$ when $R=R_{F}$. For a given $\mathrm{D}-\mathrm{A}$ pair, $R_{F}$ can be obtained by the 
usual method of overlap between the fluorescence and the absorption spectra of the D-A pair. ${ }^{24}$ For commonly used chromophores, $R_{F}$ is fairly large, often as large as $50 \AA$. This means that the rate is very large when the donor-acceptor pair is separated by a short distance. This may actually be a limitation of the Förster expression which is strictly valid when the separation between the D-A pair is much larger than their size. ${ }^{25}$

In writing Eq. (1), the standard averaging over the orientations of the transition dipole moments of the D-A pair has been carried out. In standard FRET experiments, the macromolecule is doped with a D-A pair in suitable locations along the chain. ${ }^{23}$ Excitation transfer can be monitored by following the fluorescence either from the donor or from the acceptor or from both. The time, $\tau_{r x n}$, taken for the excitation transfer to occur depends strongly on the D-A separation $R$, as given by $k(R)$ in Eq. (1). For a polymer (or a protein) in solution, both at equilibrium and during folding/ unfolding, $R$ is not only a fluctuating, stochastic function of time, but also varies in a definite way. In a recent study Wong et al. ${ }^{26}$ showed that in such cases, the distribution of the energy transfer efficiency contains nontrivial and useful information. Note that simulating FRET in real proteins is an exceedingly difficult problem-it is not trivial even for a simple homopolymer-a problem that has eluded theoretical description even today.

In this paper, we present the calculations of the distribution of FRET efficiency for Förster migration among polymer ends in various morphological structures. In the next section the calculation details of FRET efficiency distribution are presented. The model and the simulation details are described in Sec. III. Results for both the equilibrium FRET and FRET during the quenching are presented in Sec. IV. A theoretical analysis of the evaluation of structural parameters is presented in Sec. V. Conclusions are presented in Sec. VI.

\section{PROBABILITY OF FRET EFFICIENCY DISTRIBUTION}

We define the FRET efficiency $\left(\Phi_{F}\right)$ by the following relation:

$$
\Phi_{F}=\frac{k(R)}{k(R)+k_{r n}},
$$

where $k_{r n}$ is the rate which includes radiative (other than Förster migration) decay rates of the donor-acceptor pair. Note that in this study the nonradiative processes are not taken into consideration.

We next define the probability of FRET efficiency distribution $P\left(\Phi_{F}\right)$ by the following expression:

$$
P\left(\Phi_{F}\right)=\frac{1}{\mathcal{N}} \sum_{i=1}^{\mathcal{N}} \delta\left(\Phi_{F}-\Phi_{F}\left(\tau_{r x n}\right)\right) .
$$

The above equation is to be understood in the following fashion. In each simulation, after choosing a donor-acceptor pair at time $t=0$, the pair is followed till the trajectory gets terminated due to the reaction between the donor and acceptor (modeling the course of reaction in simulation is described in the next section). We denote the time taken for the trajectory to get terminated since its generation by $\tau_{r x n}$. It is important to note that in each simulation only one single polymer chain is simulated to obtain one reaction time and the corresponding donor-acceptor distance, which is then stored for the calculation of distribution of FRET efficiencies. The details of embedding the donor and acceptor into the polymer chain are described in the next section. Thus, in order to obtain a list of reaction times and their corresponding donor-acceptor distance, we had to repeat such simulations for $\mathcal{N}$ number of independent single polymer chains sampled from an equilibrium distribution. In other words, we have carried out $\mathcal{N}$ different simulation runs with independent polymer chains to obtain $\mathcal{N}$ number of $\tau_{r x n}$ and the corresponding $R$ values.

After the execution of simulation, the donor-acceptor separation $(R)$ corresponding to each reaction time is used in Eq. (2) to obtain $\Phi_{F}\left(\tau_{r x n}\right)$, which is the FRET efficiency at the corresponding time $\tau_{r x n}$. This essentially provides an array of the FRET efficiency corresponding to each reaction time obtained from simulations. A histogram of FRET efficiencies is obtained by spreading $\Phi_{F}\left(\tau_{r x n}\right)$ over a bin of width 0.1 . In this way, a continuous probability distribution $\left[P\left(\Phi_{F}\right)\right]$ can in principle be obtained from Eq. (3) by taking the $\mathcal{N} \rightarrow \infty$ limit-in our case, we get a histogram (Fig. 2). Similarly, we define the probability distribution of reaction times

$$
P\left(\tau_{r x n}\right)=\frac{1}{\mathcal{N}} \sum_{i=1}^{\mathcal{N}} \delta\left(t-\tau_{r x n}\right) .
$$

$P\left(\tau_{r x n}\right)$ can be obtained in a similar way to that of $P\left(\Phi_{F}\right)$. The only difference in this case is that the reaction times $\left(\tau_{r x n}\right)$ should be distributed instead of FRET efficiencies in to the bins accordingly to form the histogram. In this study we report only the results on FRET efficiency distribution. The results on distribution of reaction times in a similar system can be found in our earlier study. ${ }^{27}$

\section{SIMULATION DETAILS}

\section{A. The model}

The model polymer chain is made of connected Lennard-Jones (LJ) beads. This model homopolymer is known to show folding kinetics. ${ }^{1,2}$ The total potential energy of the polymer chain can be written as ${ }^{2}$

$$
U=U_{b}+U_{L J}+U_{s},
$$

where $U_{b}$ represent the bonding potential

$$
U_{b}=\sum_{i=2}^{N} b\left(\left|\mathbf{r}_{i}-\mathbf{r}_{i-1}\right|\right)^{2} .
$$

We set $b=400$ in this study. The interaction between nonbonded beads is represented by the Lennard-Jones-type potential

$$
u_{\mathrm{LJ}}(r)=\epsilon\left[\left(\frac{\sigma}{r}\right)^{12}-\left(\frac{\sigma}{r}\right)^{6}\right],
$$


where $\sigma$ and $\epsilon$ are the LJ collision diameter and the well depth, respectively. $N$ is the number of beads, $\mathbf{r}_{i}$ is the position of bead $i$, and $r_{i j}=\left|\mathbf{r}_{i}-\mathbf{r}_{j}\right|$. The stiffness is introduced through the bending potential, $U_{s}$

$$
U_{s}=\mathcal{S}(\cos \theta-1)^{2},
$$

where $\mathcal{S}$ represents the stiffness of the spring. Here, we use $N=60$, and $b=\sigma$, and change the chain stiffness by varying $\mathcal{S}$ from 1 to 10 . For convenience, we define $\epsilon^{*}=\epsilon / k_{B} T$, where $k_{B} T$ is the thermal energy. The unit of time, $\tau$, is $b^{2} / D_{0}$. Thus, $\tilde{k}_{\text {rad }}\left(\equiv k_{\text {rad }} b^{2} / D_{0}\right)$ is also dimensionless. In this study we have set $\tilde{k}_{\text {rad }}=1$. This choice of $\tilde{k}_{\text {rad }}$ corresponds to the experimentally observed $k_{\text {rad }}$ values. For example, in a solvent with viscosity $(\eta)$ equal to $1 \mathrm{cp}$, the radius of monomer molecules $(R)$ equal to $4 \AA, \tilde{k}_{\text {rad }}=1$ corresponds to a $k_{\text {rad }}$ of $0.76 \mathrm{~ns}^{27} R$ is scaled by $b$, the bead diameter, as usual. In viscous solvents, the viscosity can be much higher, and the $\tilde{k}_{\text {rad }}$ can be even larger than 10 .

The time evaluation of the polymer chain is done according to the following equation of motion: ${ }^{28,29}$

$$
\mathbf{r}_{j}(t+\Delta t)=\mathbf{r}_{j}(t)+F_{j}(t) \Delta t+\Delta X^{G}(t),
$$

where $\mathbf{r}_{j}(t)$ is the position of the $j$ th bead at time $t$, and the systematic force on $j$ is denoted by $F_{j}(t)$. The random Brownian displacement, $\Delta X^{G}(t)$, is taken from a Gaussian distribution with zero mean and $2 \Delta t$ variance. The normalized random numbers are generated by the reshuffling method. ${ }^{30}$ The time step, $\Delta t$, is chosen as $0.0005 \tau$. The scheme of Noguchi and Yoshikawa ${ }^{2}$ is used to investigate the structural transition of polymer chain. In this method, the equilibrium configuration obtained at $\epsilon^{*}=0.1$ is quenched by decreasing the temperature instantaneously to a value of $\epsilon^{*}=0.9$. The time-dependent total energy, the root-meansquare end-to-end distance $\left(R^{2}\right)$, and the radius of gyration $\left(R_{g}\right)$ were all monitored to follow the progress of collapse transition. The results presented here are the average over 10000 such trajectories with different initial configuration. More details on the simulation scheme can be found elsewhere. $^{25,31}$ To simulate FRET, we have probed many combinations of $R_{F}$ and $\tilde{k}_{\text {rad }}$. We have selected $R_{F}=6$, which is near the maximum in $R^{2} P(R)$ (we denote it by $R_{0}$ ), where $P(R)$ is the end-to-end distribution. We have also selected another value of $R_{F}$ away from the maximum in $R^{2} P(R)$, namely $R_{F}=3$. Another important parameter which affects FRET is $\tilde{k}_{\text {rad }}$. Large $\tilde{k}_{\text {rad }}$ values result in the higher efficiency of FRET. In this study we have mostly dealt with $\tilde{k}_{\mathrm{rad}}=1$.

\section{RESULTS AND DISCUSSION}

Snapshots of polymer chain with various degrees of stiffness as found in BD simulations are shown in Fig. 1. The average length of rod morphology is found to be $12.65 b$, while the average diameter of the toroid is $5.546 \mathrm{~b}$. The average diameter obtained for molten globule is $4.83 \mathrm{~b}$. We have carried out the FRET study in all three different morphologies of polymer chain and also for two different sets of Förster parameters.

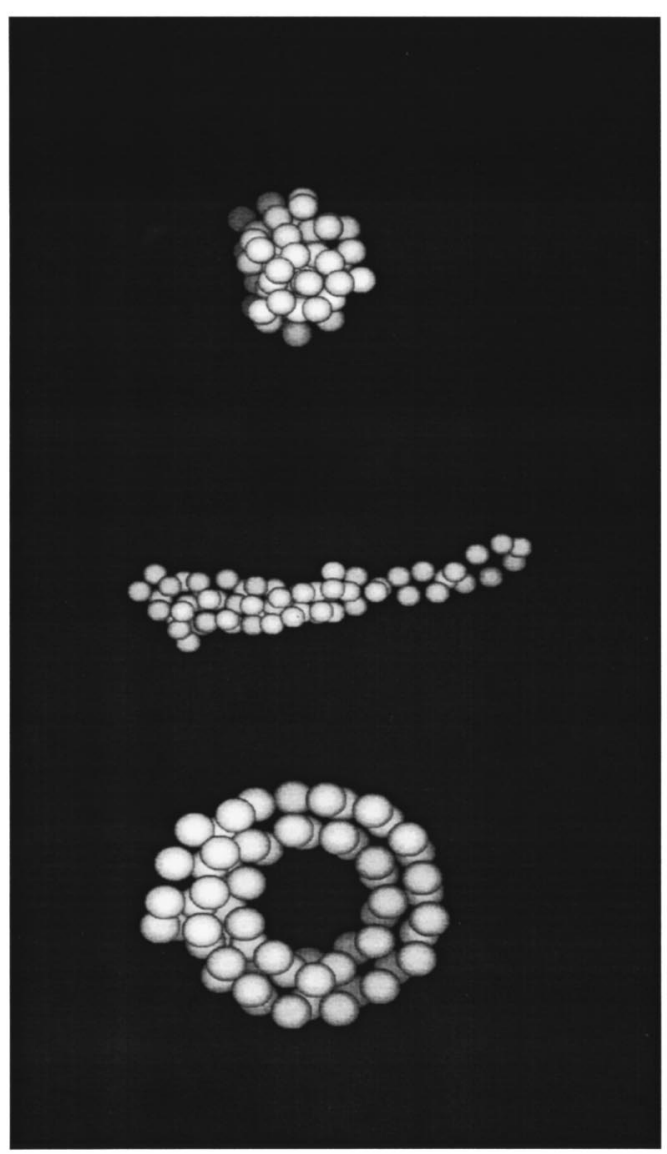

FIG. 1. Snapshots of various morphological structures of a homopolymer chain as observed in Brownian dynamics (BD) simulation for $N=60$. The chain stiffness parameter values are $\mathcal{S}=1$ (spherical), $\mathcal{S}=5$ (rod), and $\mathcal{S}=10$ (toroid) for the structures shown from top to bottom.

\section{A. Equilibrium FRET}

Morphological structures shown in Fig. 1 can be detected by monitoring the FRET in the corresponding equilibrium configuration. In order to monitor FRET in equilibrium configuration, FRET is switched on only after the polymer reaches the equilibrium configuration. This has been ensured by equilibrating the polymer for $9 \times 10^{5}$ Brownian dynamics (BD) steps. Study of the final states shows formation of various structures, depending on the chain stiffness, as already shown in Fig. 1. Results are shown in Fig. 2, where the FRET efficiency $\left[P\left(\Phi_{F}\right)\right]$ calculated by using Eq. (3) is plotted for all three different morphologies. Figures 2(a)2 (c), correspond to $\mathcal{S}=1,5$, and 10 , respectively. In all these figures $R_{F}=6$ and $k_{r n}=0.5$. Similar results are obtained at other Förster radius values. As shown in these figures, for small value of chain stiffness $P\left(\Phi_{F}\right)$ is unimodal. However, it becomes bimodal with increasing chain stiffness. The bimodal distributions for $\operatorname{rod}(\mathcal{S}=5)$ and toroid $(\mathcal{S}=10)$ resemble mirror images. Figures 2(a)-2(c) together demonstrate that the increase in chain stiffness induces a crossover in the distribution from higher efficiencies to lower efficiencies. This is also in accordance with the study of average radius of gyration $R_{g}$ for the corresponding morphological structures, as described later.

The above results can be understood in the following 

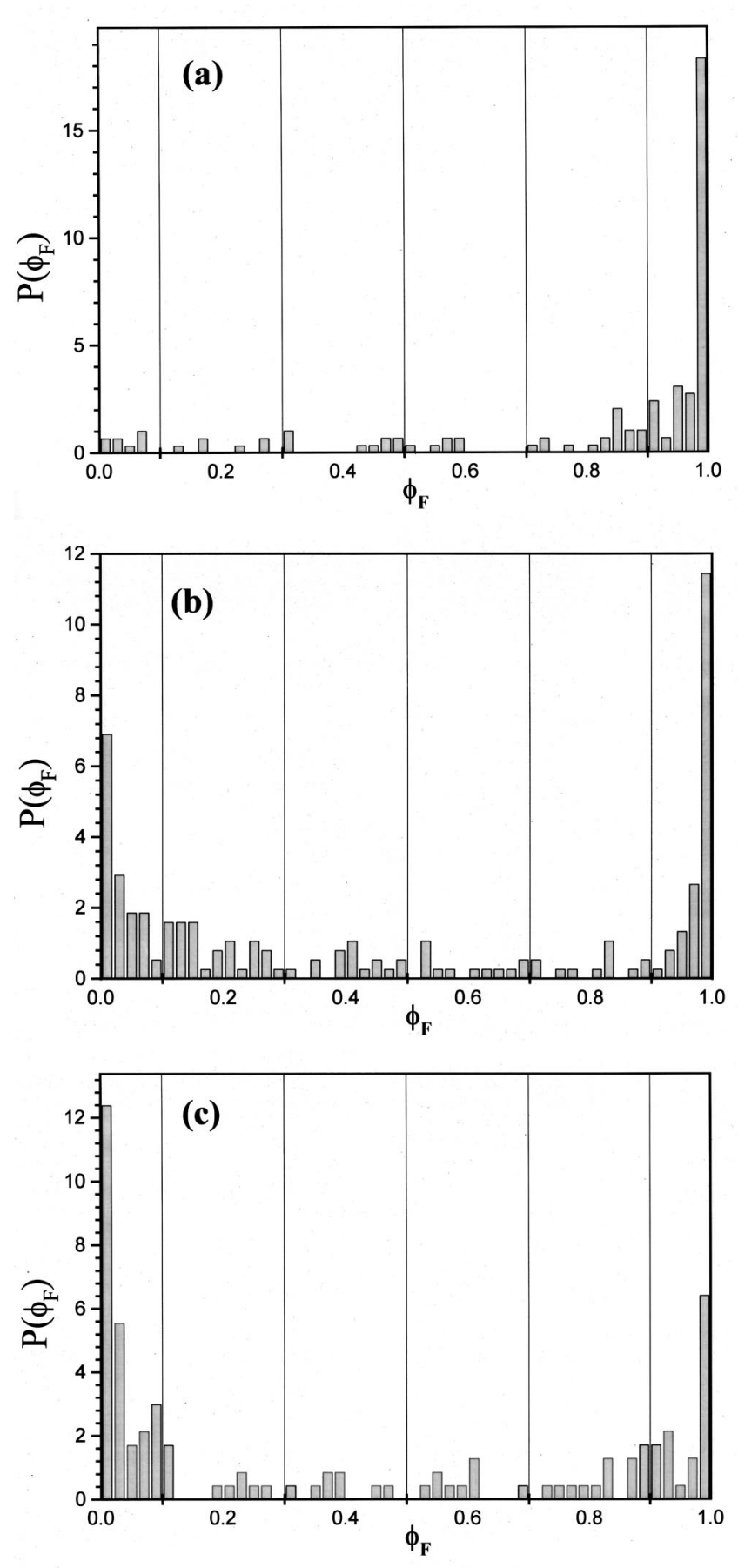

FIG. 2. The equilibrium FRET efficiency distribution $\left[P\left(\Phi_{F}\right)\right]$ obtained from BD simulations is shown for $R_{F}=6, \widetilde{k}_{\text {rad }}=1$, and $N=60$. FRET is switched on after the polymer reaches equilibrium configuration. Panels (a), (b), and (c) correspond to $\mathcal{S}=1,5$, and 10 , respectively. In all cases $k_{r n}$ $=0.5$.

manner. At lower chain stiffness value $(\mathcal{S}=1)$, the most probable morphology of the polymer chain is molten globule or spherical structure (Fig. 1). Since the end-to-end distance (or the D-A separation) is small in the molten globule state (remember that a smaller D-A separation favors FRET and vice versa), there is a large probability for the FRET to occur. Thus, in this case the FRET efficiency is large and the survival probability for the D-A pair is less. Increasing chain stiffness results in the diminishing of the peak at higher ef-
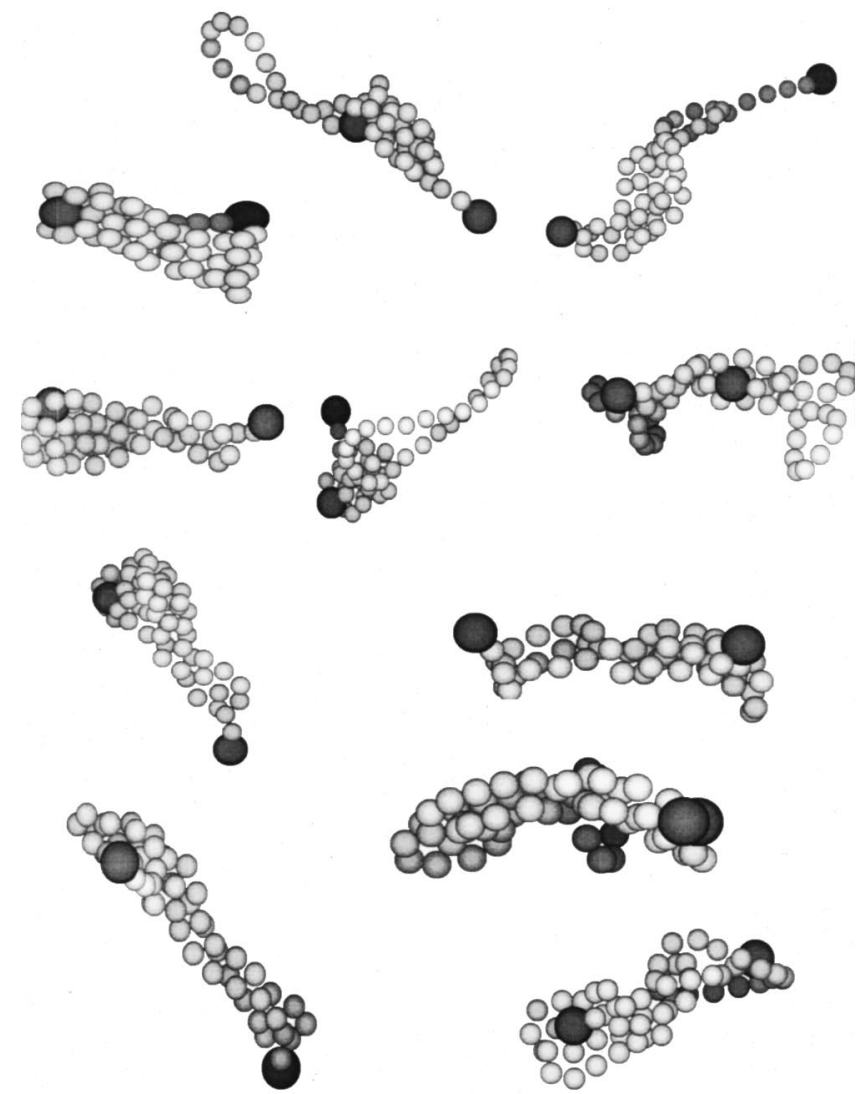

FIG. 3. Snapshots of a few rod-like conformations found in Brownian dynamics simulations, in which FRET occurred. This figure demonstrates that not only can the two ends of the chain in the rod-like conformation be close to each other, they can also be far from each other (on the opposite side).

ficiencies and also causes the emergence of a relatively broad peak at lower efficiencies [Figs. 2(b) and 2(c)]. The peak at low efficiencies corresponds to the configurations with larger D-A separation, while the one at high efficiencies results from the configurations with smaller D-A separation. The bimodality is found to depend critically on the value of $k_{r n}$, which is a consequence of several competing time scales in the FRET. The emergence of bimodal distribution suggests the existence of rod/toroidal structures with various end-toend distance. To ensure this, in Fig. 3 we have shown a few snapshots of rod conformation (as found in BD simulations) in which FRET occurred. For the sake of clarity the two end beads are shown as larger dark spheres. This figure clearly shows that not only can the two ends of the chain in the rod-like conformation be close to each other (that is, on the same side), they can also be far from each other (on the opposite side). In fact, there is a distribution of the end-toend separation even in the rod-like state. Note that there is also a distribution of the lengths of the rod which seems to indicate that a large number of polymer conformations can exist in the rod-like form. Nevertheless, the majority of the rod conformations exists with the two ends either on the same side or at the opposite side. As a result, FRET efficiency peaks both at $\Phi=1$ and $\Phi=0$ [shown in Fig. 2(b)]. Toroidal structures also give rise to a distribution, which can be understood along the same lines.

Figure 4 shows the distribution of radius of gyration at 

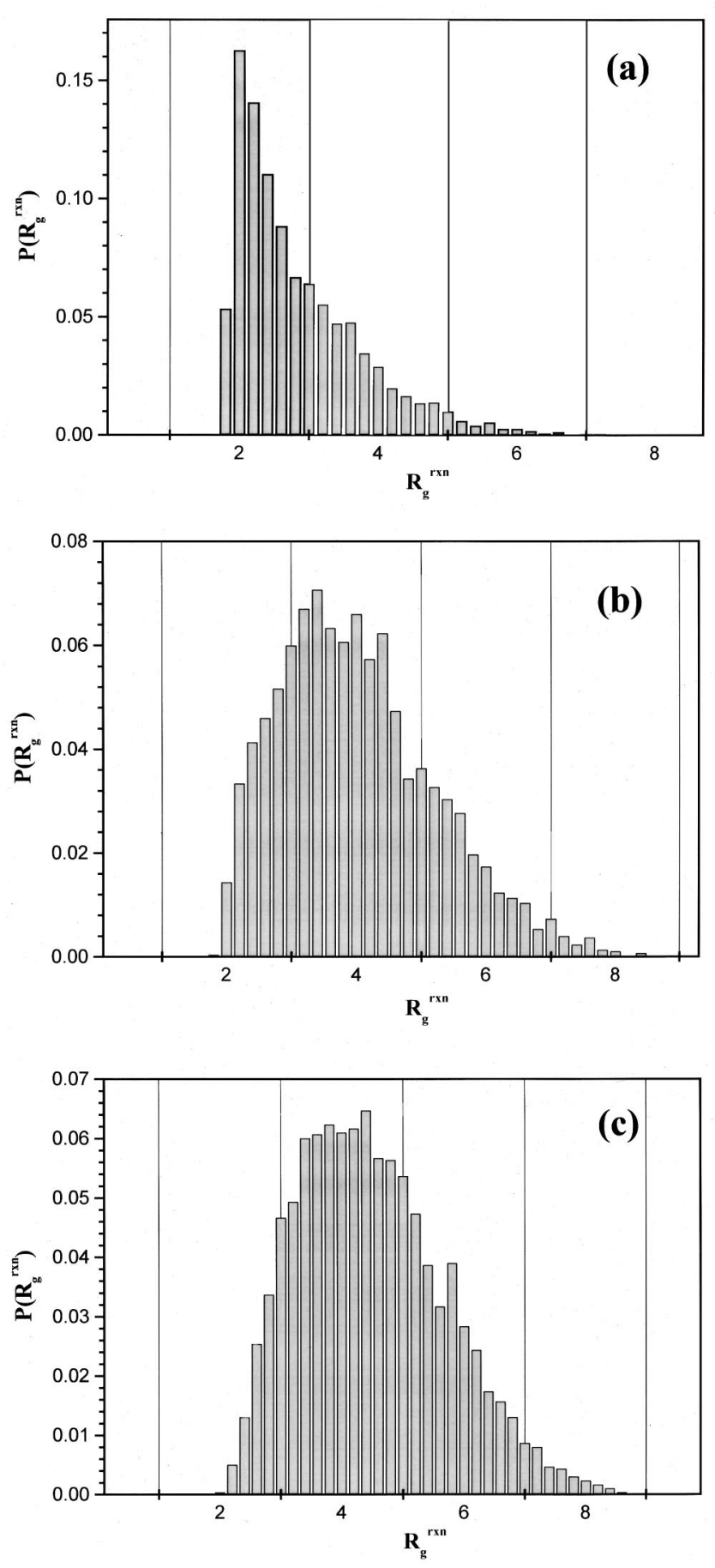

FIG. 4. The distribution of radius of gyration at which the FRET occurred is shown against $R_{g}^{r x n}$. Panels (a), (b), and (c) correspond to $\mathcal{S}=1,5$, and 10, respectively. $R_{F}=6, k_{r n}=0.5$, and $N=60$.

which the FRET occurred for various trajectories. We denote this as $R_{g}^{r x n}$ to distinguish it from $R_{g}$. Figures $4(\mathrm{a})-4(\mathrm{c})$ show the result for different stiffness values, 1, 5, and 10, respectively. In Fig. 5 the average mean-square radius of gyration obtained from BD simulation is plotted as a function of chain stiffness for two different $R_{F}$ values. Triangles and squares represent the result for $R_{F}=3$ and 6 , respectively. This figure also reveals that by decreasing the chain stiffness, polymer collapses into disordered structures and

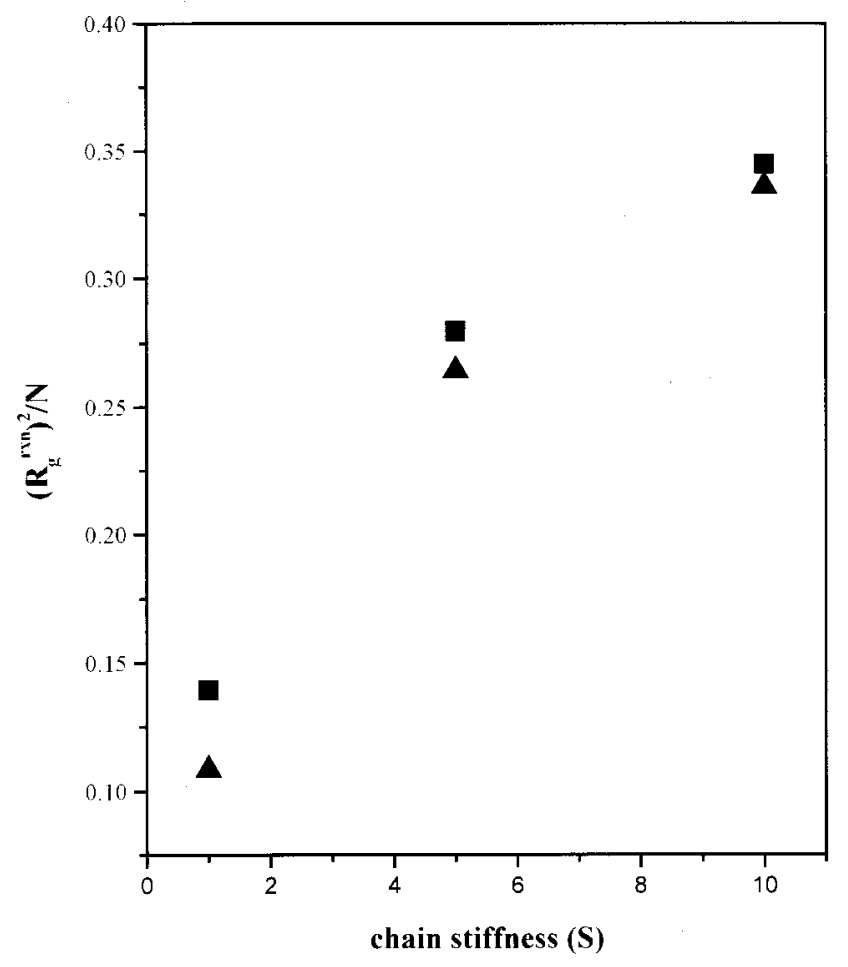

FIG. 5. The average mean-square radius of gyration at which the FRET occurred is shown as a function of chain stiffness for two different $R_{F}$ values at $k_{r n}=0.5$ for $N=60$. Filled squares represent the case with $R_{F}=6$, while the results for $R_{F}=3$ are shown by filled triangles.

FRET becomes more efficient due to the relatively smaller D-A separation.

\section{B. FRET during the quenching}

It is interesting to explore what happens if one monitors FRET during quenching. The result is shown in Fig. 6. Figure 6(a) shows the $P\left(\Phi_{F}\right)$ for $\mathcal{S}=1$, while Figs. 6(b) and 6(c) show those for $\mathcal{S}=5$ and $\mathcal{S}=10$, respectively. In this case $R_{F}$ is kept fixed at $6, k_{r n}=0.5$, and $\widetilde{k}_{\text {rad }}$ as unity. This figure reveals that in this case, while FRET can detect the spherical (or molten globule) structure, the distinction between the rod and toroidal structures is not straightforward. The reason for this can be traced to the average collapse time. For a spherical structure, collapse time is found to be much smaller than that of both rod and toroid. On the other hand, the collapse times of rod and toroid are comparable to each other. In this case, since FRET is switched on immediately after quenching, for rod and toroid conformations there is a large probability for FRET to occur before the polymer collapses into the equilibrium configuration. Thus, the FRET efficiencies observed in this way might not always correspond to the equilibrium configuration.

\section{THEORETICAL ANALYSIS: EVALUATION OF STRUCTURAL PARAMETERS}

Here, we show that the FRET efficiency distribution can be used in some cases to obtain important structural parameters, such as the "average" size of the molten globule or the average length of the rod etc. We shall assume that the 

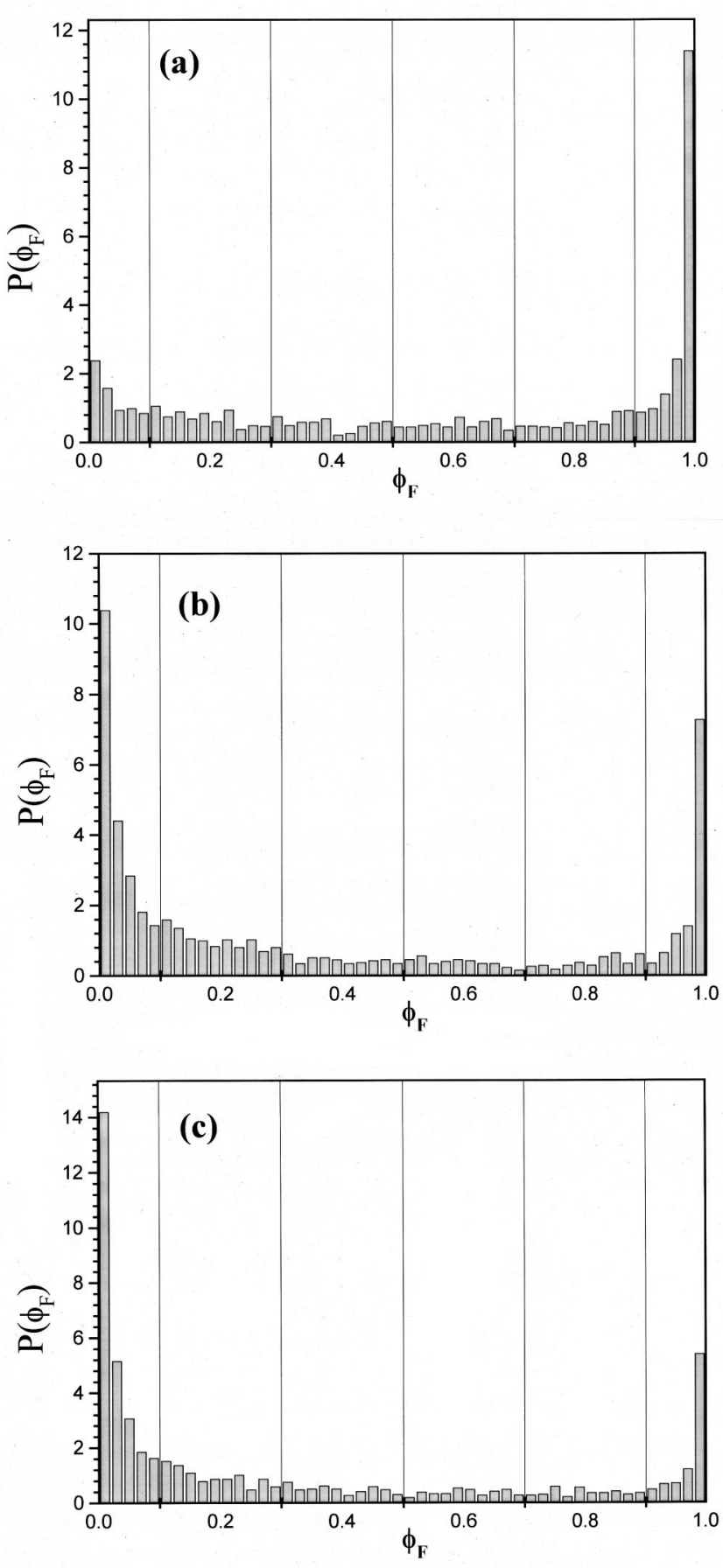

FIG. 6. The distribution of FRET efficiency $\left[P\left(\Phi_{F}\right)\right]$ during the quenching obtained from BD simulations is shown for $R_{F}=6, \tilde{k}_{\text {rad }}=1$, and $N=60$. Panels (a), (b), and (c) represent the case with $\mathcal{S}=1,5$, and 10, respectively.

Förster energy transfer occurs from an equilibrium distribution which is justified because the particular pair dies with the energy transfer. We can, therefore, define the distribution in the following way:

$$
P(\Phi)=\int d R P(R) \delta(\Phi-\Phi(R)),
$$

where $R$ is the donor acceptor separation. Note that we have defined $P(R)$ as a probability distribution of scalar $R$, which is given by

$$
P(R)=\frac{\exp (-\beta \mathcal{F}(R))}{\int d R \exp (-\beta \mathcal{F}(R))} .
$$

We next analyze the three morphological structures of polymer (molten globule, rod, and toroid) separately.

\section{A. Molten globule}

This structure is formed when chain stiffness is low. Thus, the end-to-end distribution is expected to be Gaussian, but with a force constant different from that of Rouse chain. We assume the end-to-end distribution is given by

$$
P(R)=\sqrt{\frac{3}{2 \pi\left\langle R^{2}\right\rangle}} \exp \left(-\frac{3 R^{2}}{2\left\langle R^{2}\right\rangle}\right) .
$$

In order to proceed further, next we write the Gaussian representation of the delta function

$$
\delta(\Phi-\Phi(R))=\lim _{\sigma \rightarrow 0} \frac{1}{\sigma \sqrt{(2 \pi)}} \exp \left(-\frac{(\Phi-\Phi(R))^{2}}{2 \sigma^{2}}\right) .
$$

Because of sharp variation of the Förster rate near $R_{F}$, we make the assumption that for $R<R_{F}, \Phi(R) \approx 1$ while for those $R$ greater than $R_{F}, \Phi(R) \approx 0$. This assumption allows us to write $P(\Phi)$ as the sum of two terms

$$
\begin{aligned}
P(\Phi)= & \sqrt{\frac{3}{2 \pi\left\langle R^{2}\right\rangle_{\sigma \rightarrow 0}}} \lim _{\sigma \sqrt{(2 \pi)}}\left[\int_{0}^{R_{F}} d R\right. \\
& \left.\times \exp \left(-\frac{3 R^{2}}{2\left\langle R^{2}\right\rangle}\right) \exp \left(-\frac{(\Phi(R)-1)^{2}}{2 \sigma^{2}}\right)\right] \\
& +\sqrt{\frac{3}{2 \pi\left\langle R^{2}\right\rangle_{\sigma \rightarrow 0}}} \lim \frac{1}{\sigma \sqrt{(2 \pi)}}\left[\int_{R_{F}}^{L} d R\right. \\
& \left.\times \exp \left(-\frac{3}{2} \frac{R^{2}}{\left\langle R^{2}\right\rangle}\right) \exp \left(-\frac{(\Phi(R))^{2}}{2 \sigma^{2}}\right)\right] .
\end{aligned}
$$

We can write the above equation as

$$
P(\Phi)=A_{1} \exp \left(\frac{-(\Phi-1)^{2}}{2 \sigma_{1}^{2}}\right)+\left(1-A_{1}\right) \exp \left(-\frac{\Phi^{2}}{2 \sigma_{0}^{2}}\right) .
$$

The important point here is that the coefficient $A_{1}$ will contain information about the size of the collapsed polymer. If $A_{1}$ is close to unity, as we find in Fig. 2(a), clearly the size of the polymer is less than $R_{F}$. In the results presented in Fig. $2(\mathrm{a}), R_{F}=6 b$. Thus, the size of the collapsed polymer is considerably less than $6 b$. Note that the extended Rouse chain will have $\sqrt{\left\langle R^{2}\right\rangle} \approx 7.74$ for $N=60$. For the extended chain, one expects a bimodal distribution and the values of $A_{1}$ depends on the length of the polymer chain.

For a very large polymer, it is more efficient to place the donor and the acceptor not at the ends, but some contour distance apart along the chain. This may provide better resolution. 


\section{B. Rods}

When the polymer assumes the rod-like structure at intermediate stiffness, the two ends of the chain can be uniformly separated between 0 and $L$. Thus, it is safe to assume

$$
P(R)=\frac{1}{L}, \quad L>R>0 .
$$

Therefore,

$$
P(R)=\frac{1}{L} \int_{0}^{L} d L \delta(\Phi-\Phi(L)) .
$$

We again invoke the sharp variation of $\Phi(R)$ at $R=R_{F}$ to obtain

$$
P(\Phi)=\frac{1}{L} \int_{0}^{R_{F}} d L \delta(1-\Phi)+\frac{1}{L} \int_{R_{F}}^{L} d L \delta(\Phi) .
$$

We can further coarse-grain the delta functions by their respective Gaussian distributions to obtain

$$
P(\Phi) \approx A_{1} \exp \left(\frac{-(1-\Phi)^{2}}{2 \sigma_{1}^{2}}\right)+\left(1-A_{1}\right) \exp \left(\frac{-\Phi^{2}}{2 \sigma_{0}^{2}}\right) .
$$

This is indeed a true representation that is borne out by Fig. 2(b). In the present case, however, we can obtain an expression for $A_{1}$

$$
A_{1}=\frac{R_{F}}{L \sigma_{1}} \sqrt{\frac{2}{\pi}}
$$

which is needed to obtain the length $L$. From Fig. 2(b) we find $A_{1} \approx 11.5, \sigma_{1} \approx 0.03$, and since $R_{F}=6$, we get average $L=13.87 b\left(\approx 2 R_{F}\right)$, which is in good agreement with simulations $(12.65 b)$.

An alternative way is to argue that the total amount near $\Phi=1$ is equal to $R_{F} / L$. Since this amount is about 0.5 [see Fig. 2(b)], this again gives a value of $L \approx 12 b$. Therefore, one can use the FRET efficiency distribution, $P(\Phi)$, to obtain an estimate of the length of the rod morphology.

\section{Toroid}

We can carry out a simple analysis in the same spirit as above to obtain an estimate of the average radius of the toroid, $R_{\text {tor }}$. While for accurate analysis one needs to employ the equation of the toroid, here we shall employ a simpler one based on the assumption that the two ends can, with equal probability, be anywhere between 0 and $2 R_{\text {tor }}+\Delta$, where $\Delta$ is the thickness of the toroid. Thus, we can write

$$
P(R)=\frac{1}{\left(2 R_{\text {tor }}+\Delta\right)} .
$$

Then, an analysis similar to the one performed above leads to the following expression for $R_{\text {tor }}$ :

$$
R_{\text {tor }}+\Delta=\frac{R_{F}}{2 A_{1} \sigma_{1}} \sqrt{\frac{2}{\pi}} .
$$

From Fig. 2(c), we find $A_{1} \approx 6, \sigma_{1} \approx 0.07, \Delta \approx 3$, and thus, average $R_{\text {tor }} \approx 3.9 b$, which is in agreement with the simulation result $(2.77 b)$.
The above analyses are only for the case where the $\mathrm{D}-\mathrm{A}$ pair is located at the two ends of the polymer chain. The analysis is particularly crude for toroid where one should consider the equation for the toroid.

\section{CONCLUSION}

In this work we have shown that the FRET can serve as a method to obtain information on the various conformations of a homopolymer chain. The distribution of excitation energy transfer efficiency is unimodal for low chain stiffness values, but becomes bimodal with increasing the chain stiffness. The extent of bimodality is found to depend on the values of the Förster parameters $\left(k_{\text {rad }}\right.$ and $\left.R_{F}\right), k_{r n}$, and also the value of the diffusion coefficient (that is, the viscosity). The theoretical analyses also reveal that one can use the FRET efficiently distribution to obtain information about the various morphological structures of polymers.

The present study reveals that while equilibrium FRET can be used to detect various collapsed structures, FRET during the quenching might not do the same. Thus, a study like the one performed here can be useful in designing FRET experiments via single molecule spectroscopy to carry out the conformational studies of polymers. The present study suggests several exciting future possibilities. Simulation study by using more realistic models and also by incorporating the solvent molecules explicitly will certainly reveal more information. Another useful solution is to develop theoretical models to understand the energy transfer efficiency distribution along the lines recently initiated by Wolynes and co-workers. ${ }^{32-35}$ Further work in these directions is in progress.

\section{ACKNOWLEDGMENTS}

The financial support from CSIR, New Delhi, India and DST India is gratefully acknowledged. One of the authors (G.S.) thanks CSIR for a research fellowship.

${ }^{1}$ D. Hu, J. Yu, K. Wong, B. Bagchi, P. Rossky, and P. F. Barbara, Nature (London) 405, 1030 (2000).

${ }^{2}$ H. Noguchi and K. Yoshikawa, J. Chem. Phys. 113, 854 (2000); 109, 5070 (1998); Chem. Phys. Lett. 278, 184 (1997).

${ }^{3}$ Y. A. Kuznestov and E. G. Timoshenko, J. Chem. Phys. 111, 3744 (1999).

${ }^{4}$ V. A. Ivanov, W. Paul, and K. Binder, J. Chem. Phys. 109, 5659 (1998).

${ }^{5}$ V. A. Ivanov, M. R. Stukan, V. V. Vasilevskaya, W. Paul, and K. Binder, Macromol. Theory Simul. 9, 488 (2000).

${ }^{6}$ S. T. Sun, I. Nishio, G. Swislow, and T. Tanaka, J. Chem. Phys. 73, 5971 (1980).

${ }^{7}$ H. Noguchi, S. Saito, S. Kidoaki, and K. Yoshikawa, Chem. Phys. Lett. 261, 527 (1996).

${ }^{8}$ A. Yekta, J. G. Spiro, and M. A. Winnik, J. Phys. Chem. B 102, 7960 (1998).

${ }^{9}$ M. A. Winnik, Macromolecules 30, 2633 (1997).

${ }^{10}$ A. Baumgartner, in Applications of the Monte Carlo Method in Statistical Physics, 2nd ed., edited by K. Binder (Springer, New York, 1987).

${ }^{11}$ G. Srinivas, A. Yethiraj, and B. Bagchi, J. Phys. Chem. B 105, 2475 (2001).

${ }^{12}$ M. Iwata and H. Sato, Phys. Chem. Chem. Phys. 1, 2491 (1999).

${ }^{13}$ A. Berg, Int. J. Mod. Phys. C 3, 1083 (1992).

${ }^{14}$ K. F. Wong, M. S. Skaf, C.-Y. Yang, P. J. Rossky, B. Bagchi, D. Hu, J. Yu, and P. F. Barbara, J. Phys. Chem. B 105, 6103 (2001).

${ }^{15} \mathrm{Ph}$. Tamarat, A. Maali, B. Lounis, and M. Orrit, J. Phys. Chem. A 104, 1 (2000) 
${ }^{16}$ A. A. Deniz, T. A. Laurence, G. S. Beligere, M. Dahan, A. B. Martin, D. S. Chemla, P. E. Dawson, P. G. Schultz, and S. Weiss, Proc. Natl. Acad. Sci. U.S.A. 97, 5179 (2000).

${ }^{17}$ A. A. Deniz, M. Dahan, J. R. Grunwell, T. Ha, A. E. Faulhaber, D. S. Chemla, S. Weiss, and P. G. Schultz, Proc. Natl. Acad. Sci. U.S.A. 96, 3670 (1999).

${ }^{18}$ S. Weiss, Science 283, 676 (1999).

${ }^{19}$ D. S. Talaga, W. L. Lau, H. Roder, J. Tang, W. F. De Grado, and R. M. Hoschstrasser, Proc. Natl. Acad. Sci. U.S.A. 97, 13021 (1999).

${ }^{20}$ M. I. Wallace, L. Ying, S. Balasubramanian, and D. Klenermen, J. Phys. Chem. B 104, 11551 (2000); 104, 5171 (2000).

${ }^{21}$ G. S. Jas, W. A. Eaton, and J. Hofrichter, J. Phys. Chem. B 105, 261 (2001).

${ }^{22}$ Th. Förster, Ann. Phys. (Leipzig) 2, 55 (1948).

${ }^{23}$ L. Stryer and R. P. Haugland, Proc. Natl. Acad. Sci. U.S.A. 58, 719 (1967).
${ }^{24}$ C. R. Cantor and P. Pechukas, Proc. Natl. Acad. Sci. U.S.A. 68, 2099 (1971).

${ }^{25}$ G. Srinivas and B. Bagchi, J. Phys. Chem. B 105, 9370 (2001).

${ }^{26}$ K. F. Wong, C.-Y. Yang, B. Bagchi, and P. J. Rossky (unpublished).

${ }^{27}$ G. Srinivas and B. Bagchi, Chem. Phys. Lett. 328, 420 (2000).

${ }^{28}$ R. W. Pastor, R. Zwanzig, and A. Szabo, J. Chem. Phys. 105, 3878 (1996).

${ }^{29}$ D. L. Ermak and J. A. McCammon, J. Chem. Phys. 69, 1352 (1978).

${ }^{30}$ W. H. Press, S. A. Teulolsky, W. T. Vellerling, and B. P. Flannery, Numerical Recipes in FORTRAN (Cambridge University Press, Cambridge, 1986).

${ }^{31}$ G. Srinivas, A. Yethiraj, and B. Bagchi, J. Chem. Phys. 114, 9170 (2001).

${ }^{32}$ J. J. Portman, S. Takada, and P. G. Wolynes, J. Chem. Phys. 114, 5069 (2001); 114, 5082 (2001).

${ }^{33}$ J. J. Portman and P. G. Wolynes, J. Phys. Chem. A 103, 10602 (1999).

${ }^{34}$ J. Wang and P. G. Wolynes, J. Chem. Phys. 110, 4812 (1999); Phys. Rev. Lett. 74, 4317 (1995).

${ }^{35}$ N. J. Turro, Modern Molecular Photochemistry (Benjamin, Cummings, CA, 1978). 